Results 233 children were followed for a median of 2.0 (1.7$2.3)$ years. The median (IQR) age was $8.0(5.0-12.2)$ years. 71 children isolated PA during the study period. The incidence rate (95\% CI) of new PA infections was $0.15(0.10-0.22)$ cases per patient year for those that had never previously isolated PA and $0.19(0.13-0.27)$ cases per patient year for those that had been free from PA for $>12$ months. This rate varied between 0.08 $(0.04-0.18)$ and $0.28(0.14-0.49)$ cases per patient year at the 8 recruiting centres. $42 \%$ of children were asymptomatic at the time of PA acquisition. The median (IQR) number of antibiotic courses per patient year varied between the centres: $0.6(0.2-$ $1.3)$ to $3.6(3.1-4.2)$ for oral and $0.0(0-0)$ to $0.4(0-1.2)$ for intravenous.

Conclusions This is the first prospective study to report the incidence of new PA infection in a large cohort of children with CF, considered to be free of PA airway infection. Incidence rate was higher in children who had isolated PA previously. The variation between centres is not easily explained and needs further investigation.

Acknowledgments We would like to thank the Principal Investigators, research nurses and co-ordinators at each of the recruiting centres as well as the children and their families.

\section{P198 NEW APPROACHES TO THE CULTURE OF MYCOBACTERIUM ABSCESSUS COMPLEX FROM PATIENTS WITH CYSTIC FIBROSIS}

${ }^{1} \mathrm{JE}$ Foweraker, 'S Jalili, 'V Athithan, ${ }^{1} \mathrm{D}$ Grogono, ${ }^{2} \mathrm{MC}$ Curran, ${ }^{1} \mathrm{RA}$ Floto. ${ }^{1}$ Papworth Hospital NHS Foundation Trust, Cambridge, UK; ${ }^{2}$ Cambridge Clinical Microbiology and Public Health Laboratory, Cambridge, UK

\subsection{6/thoraxjn-2014-206260.327}

Introduction M.abscessus complex (Mab) are Rapid Growing Mycobacteria (RGM) that can cause severe infection. Prevalence is increasing and a recent study using whole genome sequencing showed cross infection between Cystic Fibrosis patients. Frequent surveillance for Mab infection may allow earlier diagnosis and prevent spread.

Automated broth (e.g. MGIT), is a sensitive rapid method for mycobacterial culture. Decontamination is needed to kill other bacteria and yeasts before culturing CF sputa in MGIT, but decontamination may reduce Mab numbers.

Other possibilities include chlorhexidine decontamination which yields more Mab but is incompatible with MGIT. Some mycobacteria grow directly from sputum on Burkholderia cepacia selective agar $(\mathrm{Bcc})$ after extended incubation, without prior decontamination.

The aim of this study was to improve Mab culture from CF sputum.

Methods We compared MGIT culture of CF sputa with extended incubation of Bcc used in the routine laboratory. We compared growth of 30 known Mab on 3 formulations of Bcc and 2 Middlebrooke selective agars. We took 12 sputa from 9 CF patients with Mab infection and compared MGIT with culture on selective agars or chlorhexidine decontamination followed by culture onto non selective agar. Mycobacteria were identified by the National Mycobacterium Reference Laboratory and an in house PCR.

Results Eighteen of 515 CF sputa grew RGM (9 on Bcc agar and MGIT, 3 MGIT alone, $4 \mathrm{Bcc}$ alone and 2 on Bcc with no MGIT culture). Contamination with other bacteria and fungi made it extremely difficult to see RGM on the routine Bcc.
Thirty sequenced M.abscessus abscessus, M.bolletii and M. massilliense all grew on the 3 commercial Bcc and 2 Middlebrooke agars.

One Bcc and one Middlebrooke agar successfully cultured RGM from all 12 sputa with fewest contaminants. Chlorhexidine decontamination and blood agar was effective but labourintensive. Only 8 of 12 MGIT cultures grew RGM.

There was no difference in time to positive culture between agar and MGIT.

Conclusion Culture onto selective agar may be more sensitive than MGIT. It is quantitative and provides pure culture for identification, typing and susceptibility testing.

This may be a sensitive cost-effective way to screen sputa from patients at risk.

\section{P199 MOLECULAR ANALYSIS DEMONSTRATES SHARED STRAINS OF MYCOBACTERIUM ABSCESSUS ISOLATES IN CYSTIC FIBROSIS PATIENTS ATTENDING A SINGLE CENTRE}

${ }^{1} \mathrm{HD}$ Green, ${ }^{1}$ Rowland Bright-Thomas, ${ }^{1} \mathrm{PJ}$ Barry, ${ }^{2} \mathrm{~N}$ Woodford, ${ }^{3} \mathrm{~B}$ Isalska, ${ }^{1} \mathrm{~A}$ Horsley, ${ }^{2} \mathrm{D}$ Kenna, ${ }^{1}$ AM Jones. ${ }^{1}$ Manchester Adult Cystic Fibrosis Centre, University Hospital of South Manchester, Manchester, UK; ${ }^{2}$ Public Health England, Antimicrobial Resistance and Healthcare Associated Infections Reference Unit, London, UK; ${ }^{3}$ Clinical Microbiology, University Hospital of South Manchester, Manchester, UK

\subsection{6/thoraxjnl-2014-206260.328}

Introduction and objectives The Mycobacterium abscessus complex is an emerging group of pathogens in cystic fibrosis (CF), which may cause cross infection. The aim of this study was to determine whether CF patients infected with M.abscessus attending a single UK adult CF centre harboured unique or shared strains of M. abscessus.

Methods Isolates were from 12 patients attending a single adult CF centre, who yielded one or more positive sputum cultures for M. abscessus complex during the period January 2010 to August 2013. Isolates were identified to subspecies level using hsp65-rpoB concatenated sequence cluster analysis. Variable Number Tandem Repeat (VNTR) analysis was used to compare these isolates and determine whether two or more patients were infected with the same strain.

Results 11 isolates were identified as M. abscessus susbp. abscessus. VNTR analysis demonstrated 2 clusters of 6 and 2 patients carrying the same strains of $M$. abscessus susbp. abscessus, both

\begin{tabular}{|c|c|c|}
\hline Patient & M.abscessus subspecies & VNTR Profile \\
\hline 1 & abscessus & $2+, 5+, 3,6,2,5,1+, 2+$, \\
\hline 2 & abscessus & $2+, 5+, 3,6,2,5,1+, 2+,-$ \\
\hline 3 & abscessus & $2+, 5+, 3,6,2,5,1+, 2+,-$ \\
\hline 4 & abscessus & $2+, 5+, 3,6,2,5,1+, 2+,-$ \\
\hline 5 & abscessus & $2+, 5+, 3,6,2,5,1+, 2+,-$ \\
\hline 6 & abscessus & $2+, 5+, 3,6,2,5,1+, 2+,-$ \\
\hline 7 & abscessus & $3+, 4+, 3,-4,3+, 2+, 2+, 2$ \\
\hline 8 & abscessus & $3+, 4+, 3,2,4,3+, 2+, 2+, 2$ \\
\hline 9 & abscessus & $2+,-, 3,4,2,5,1+, 2+,-$ \\
\hline 10 & abscessus & $-5+, 2,2+, 4,5,1+, 3,2$ \\
\hline 11 & abscessus & $1+, 4+, 2,2,4,3+, 2+, 2+, 2$ \\
\hline 12 & bolettii & $2+,-, 5,5,4,3+, 1+, 2,1+$ \\
\hline
\end{tabular}


of which have also been isolated from CF patients from other UK hospitals. Isolates from the remaining 3 patients were unique. One additional isolate was identified as M. abscessus subsp.bolletii. No clear epidemiological connexions between patients within each cluster at our centre have been identified to date.

Conclusion These results provide further evidence that some strains of M. abscessus complex may be isolated from multiple CF patients. However, there were no clear epidemiological connexions between patients within clusters at our centre. The same strains have been isolated from patients at different UK CF centres. Further studies are required to determine the mode of acquisition of infection with these strains and whether there is a common environmental source of infection or cross infection between patients.

\section{P200 PRELIMINARY EVALUATION OF THE FUNGAL AIRWAY MICROBIOME IN ADULT CYSTIC FIBROSIS BY NEXT- GENERATION SEQUENCING, CULTURE AND STAINING TECHNIQUES}

${ }^{1} \mathrm{IC}$ Felton, ${ }^{1} \mathrm{~S}$ Benson, ${ }^{1} \mathrm{~A}$ Nicholson, ${ }^{1} \mathrm{~K}$ Al-Shafi, ${ }^{2} \mathrm{P}$ James, ${ }^{2} \mathrm{MJ}$ Cox, ${ }^{2} \mathrm{AJ}$ Walley, ${ }^{2} \mathrm{MF}$ Moffatt, ${ }^{1} \mathrm{D}$ Bilton, ${ }^{1} \mathrm{MR}$ Loebinger, ${ }^{1} \mathrm{NJ}$ Simmonds, ${ }^{2}$ WO Cookson. ${ }^{1}$ Royal Brompton and Harefield NHS Foundation Trust, London, UK; ${ }^{2}$ National Heart and Lung Institute, Imperial College London, London, UK

\subsection{6/thoraxjnl-2014-206260.329}

Introduction The prevalence and diversity of fungal airway isolates is increasing in cystic fibrosis (CF). Amidst an extending spectrum of fungal complications, lack of standardised mycology methods and poor sensitivity of culture-dependent techniques renders interpretation of isolates challenging.

Aims To evaluate the diagnostic utility of fungal cytology and microbiology stains in addition to prolonged sputum culture from adult CF patients in comparison to standard mycology techniques.

Secondly, to develop a novel, next-generation sequencing assay targeting the ITS2 region of the fungal ribosomal-RNA gene to comprehensively profile the sputum fungal microbiota.

Methods Sputum samples were investigated by a panel of three mycology techniques: prolonged fungal culture (each examined at: Day7, D14, D21, D28); Calcofluor White (CFW) stain; Grocott's Methenamine Silver (GMS) stain. A cohort of samples was also subject to broad-spectrum fungal next-generation sequencing.

Results 25 adult patients provided 45 sputum samples. Four fungal species were cultivatable: Candida species (26.6\%); Aspergillus fumigatus (4.4\%); Scedosporium apiospermum (15.5\%) and Exophiala dermatitidis (11.1\%).

Prolonged culture significantly increased overall fungal prevalence by $22 \%$ compared to standard duration (D7) $\mathrm{p}=0.008$ ). A significant increase of $11.1 \%$ in $S$. apiopspermum prevalence was observed $\mathrm{p}=0.02$ ), whilst all $E$. dermatitidis isolates required prolonged culture. The sensitivity of GMS and CFW stains $(85 \%$ and $93 \%)$ compared favourably to standard duration culture (29\%).

DNA extracted from a pilot group of these sputum samples ( $n=14 / 45)$ was subject to PCR using barcode-indexed ITS2 primers designed for Illumina-MiSeq amplicon sequencing. Fungal taxa were detected in all samples, of which seven samples (50\%) were negative after prolonged culture. Preliminary sequencing analysis of an extended sample cohort $(n=30)$ has detected 89 fungal taxa, from which only four species were cultured.
Conclusions Prolonged fungal culture is associated with a significant increase in fungal prevalence. The increased sensitivity is restricted to less common filamentous fungi associated with increasing pathogenicity: S. apiospermum and E. dermatitidis. The predictive value of stains in identifying samples positive at prolonged culture, but negative at standard duration illustrates their clinical utility.

Illumina-MiSeq ITS2-amplicon sequencing directly from sputum has identified a more diverse CF airways fungal microbiota. Preliminary analysis suggests that this is a highly sensitive tool for detecting fungi from sputum, including species which are refractory to standard and enhanced culture.

\section{P201 PNEUMOCYSTIS JIROVECII PREVALENCE IN A LARGE UK ADULT CYSTIC FIBROSIS CENTRE}

${ }^{1} \mathrm{HD}$ Green, ${ }^{1} \mathrm{R}$ Bright-Thomas, ${ }^{1} \mathrm{PJ}$ Barry, ${ }^{1} \mathrm{~A}$ Horsley, ${ }^{2} \mathrm{~K}$ Mutton, ${ }^{1} \mathrm{AM}$ Jones. ${ }^{1}$ Manchester Adult Cystic Fibrosis Centre, University Hospital of South Manchester, Manchester, UK; ${ }^{2}$ Clinical Virology Department, Central Manchester University Hospitals NHS Trust, Manchester, UK

\subsection{6/thoraxjnl-2014-206260.330}

Introduction and objectives Pneumocystis jirovecii $(P J)$ is an atypical fungus that causes pneumonia in immunocompromised patients. Its role in patients with cystic fibrosis $(\mathrm{CF})$ is unclear. Its reported prevalence in CF ranges from 1-22\% but has never been determined in the UK. Here we present preliminary crosssectional data from an ongoing study at a UK adult CF centre

Abstract P201 Table 1 Comparison of characteristics for patients with positive and negative samples

\begin{tabular}{|c|c|c|c|}
\hline \multicolumn{4}{|l|}{ Pneumocystis Jiroveccii result } \\
\hline & Negative & Positive & Sig. \\
\hline Number & 93 & 7 & NS \\
\hline \multicolumn{4}{|l|}{ Demographics } \\
\hline Male gender, n (\%) & $52(55.9)$ & $4(57.1)$ & NS \\
\hline Mean age (yr), SD & $33.8 \pm 11.1$ & $32.5 \pm 10.1$ & NS \\
\hline \multicolumn{4}{|c|}{ Spirometry and laboratory results } \\
\hline Mean $\mathrm{FEV}_{1}$ (\% predicted), SD & $56 \pm 20.1$ & $67 \pm 29.5$ & NS \\
\hline Mean BMI $\left(\mathrm{kg} / \mathrm{m}^{2}\right)$ & $22.1 \pm 3.2$ & $22.2 \pm 2.1$ & NS \\
\hline WCC $\left(x 10^{9} / \mathrm{L}\right)$ at recruitment & $9.3 \pm 3.1$ & $9.3 \pm 2.8$ & NS \\
\hline CRP (mg/L) at recruitment & $13.0 \pm 16.8$ & $7.9 \pm 7.5$ & NS \\
\hline \multicolumn{4}{|l|}{ Chronic colonisation, n (\%) } \\
\hline Pseudomonas aeruginosa & $59(63.4)$ & $5(71.4)$ & NS \\
\hline Staphylococcus aureus & $24(25.8)$ & $1(14.3)$ & NS \\
\hline B. cepacia complex & $14(10.3)$ & $2(28.6)$ & NS \\
\hline Achromobacter xylosoxidans & $6(6.4)$ & 0 & NS \\
\hline Ralstonia species & $5(5.4)$ & 0 & NS \\
\hline NTM & $5(5.4)$ & $1(14.3)$ & NS \\
\hline Aspergillus fumigatus & $4(4.3)$ & $1(14.3)$ & NS \\
\hline Pandoraea species & $1(1.1)$ & $1(14.3)$ & NS \\
\hline MRSA & $1(1.1)$ & $1(14.3)$ & NS \\
\hline \multicolumn{4}{|l|}{ Treatment, n (\%) } \\
\hline Inhaled antibiotics & $72(77.4)$ & $6(85.7)$ & NS \\
\hline Inhaled steroids & $59(63.4)$ & $6(85.7)$ & NS \\
\hline Oral steroids (continuous) & $12(12.9)$ & $1(14.3)$ & NS \\
\hline Prophylactic macrolide & $77(83.0)$ & $5(71.4)$ & NS \\
\hline Azole therapy & $8(8.6)$ & 0 & NS \\
\hline Co-trimoxazole past 3 months & $32(34.4)$ & 0 & 0.06 \\
\hline
\end{tabular}

Key: $\mathrm{SD}=$ standard deviation, $\mathrm{WCC}=$ serum white cell count, $B$.cepacia complex $=$ Burkholderia cepacia complex, NTM $=$ Non-tuberculous mycobacteria, MRSA $=$ Methicillin resistant Staphylococcus aureus, NS $=$ not significant, $\mathrm{p}$ values $<0.1$ are shown. 